\title{
MRSA-Screening in der neurologisch-neurochirurgischen Frührehabilitation
}

\section{MRSA screening in early neurological and neurosurgical rehabilitation}

\author{
Autoren \\ Jens D. Rollnik', Ludwig Grüter² \\ Institute \\ 1 BDH-Klinik Hessisch Oldendorf gGmbH, Institut für \\ neurorehabilitative Forschung (InFo), Medizinische \\ Hochschule Hannover \\ 2 Nordlab, Hameln \\ Schlüsselwörter \\ MRSA, PCR, Kultur, Sensitivität, Spezifität
}

Key words

MRSA, PCR, culture, sensitivity, specificity

eingereicht 18.03.2019

akzeptiert 29.03.2019

Bibliografie

DOI https://doi.org/10.1055/a-0889-8163

Fortschr Neurol Psychiatr 2019; 87: 499-502

(c) Georg Thieme Verlag KG Stuttgart · New York

ISSN 0720-4299

Korrespondenzadresse

Prof. Jens D. Rollnik

BDH-Klinik Hessisch Oldendorf gGmbH, Institut für

neurorehabilitative Forschung (InFo), Medizinische

Hochschule Hannover

Greitstr. 18-28

31840 Hessisch Oldendorf

Tel.: 05152781231

Fax: 05152781198

E-Mail: prof.rollnik@bdh-klinik-hessisch-oldendorf.de

\section{ZUSAMMENFASSUNG}

Methicillin-resistente Staphylococcus-aureus-Stämme (MRSA) haben in der neurologisch-neurochirurgischen Frührehabilitation eine erhebliche medizinische und ökonomische Relevanz. Es ist wichtig, MRSA-Träger möglichst rasch nach Aufnahme zu identifizieren, um Transmissionen zu verhindern und Isolierungssowie Dekolonisierungsmaßnahmen einleiten zu können. In der vorliegenden Arbeit wurde die Anwendung und Aussagekraft eines PCR-basierten Schnelltests (Polymerase-Kettenreaktion) zum Nachweis von MRSA-Erbgut in Nasen-Rachen-Abstrichen (BD MAX MRSA XT, BD Diagnostics, Heidelberg, Deutschland) von neurologischen Rehabilitanden untersucht und mit dem Standard-Verfahren (Kultur) als Goldstandard verglichen. Im Jahr 2013 wurden innerhalb von sieben Tagen nach Aufnahme bei $\mathrm{n}=66$ Frührehabilitanden MRSA-Abstriche sowohl mittels PCRSchnelltest als auch kulturell untersucht. Dabei ergab sich für die PCR eine Sensitivität von 84,6\% mit einer Spezifität von $86,8 \%$. Der positive prädiktive Wert (PPW) lag bei nur $61,1 \%$, der negative (NPW) bei 95,8\%. Bei insgesamt 39 Frührehabilitanden lagen PCR und Kultur zeitlich nur bis zu einem Tag auseinander, hierdurch stieg die Sensitivität auf $100 \%$, die Spezifität auf $90,3 \%$. In dieser Subgruppe betrug der PPW 72,7\%, der NPW $100 \%$. Die Ergebnisse zeigen, dass nach einem positiven PCR-Befund rasch (innerhalb von 24 Stunden) eine kulturelle Untersuchung durchgeführt werden sollte, um eine MRSABesiedelung zu verifizieren. Insbesondere der hohe NPW (95,8 bzw. $100 \%$ ) zeigt an, dass negativ getestete Patienten auch mit hoher Wahrscheinlichkeit keine MRSA-Träger sind. Auf ein positives Testergebnis in der PCR kann man sich allerdings weniger gut verlassen (wegen falsch-positiver Ergebnisse), sodass bei Vorliegen eines MRSA-Nachweises in der PCR bei neurologischneurochirurgischen Frührehabilitanden eine kulturelle Untersuchung durchgeführt werden sollte, um unnötig lange Isolierungsmaßnahmen zu vermeiden.

\section{ABSTRACT}

Strains of methicillin-resistant Staphylococcus aureus (MRSA) are of major economic and health-related importance to early neurological and neurosurgical rehabilitation. It is crucial to identify MRSA-carriers as soon as possible upon admission in order to prevent transmissions and to initiate contact precautions and decolonization. The present study focuses on validity of a polymerase chain reaction (PCR) test to identify MRSA genetic material from nasopharyngeal samples (BD MAX MRSA XT, BD Diagnostics, Heidelberg, Germany) of early neurological and neurosurgical rehabilitation patients. PCR-results were compared to gold standard (culture). In 2013, 66 patients were tested using PCR and incubation within one week after admission. Sensitivity of PCR was $84.6 \%$, specificity $86.6 \%$. Positive predictive value (PPV) was only $61.1 \%$, while negative predictive value was as high as $95.8 \%$. In 39 cases, PCR and subsequent culture were done within one day, leading to a sensitivity of $100 \%$ and a specificity of $90.3 \%$. In this subgroup, 
PPV was $72.7 \%$, NPV $100 \%$. The results from the study suggest that incubation should quickly follow a positive PCR finding (within 24 hours) in order to verify MRSA colonization. High NPV (95.8 resp. $100 \%$ ) indicate that PCR negative patients very likely are not colonized with MRSA. A positive PCR test is less reliable (due to false positive results) and should be followed by incubation in due course in order to avoid unnecessary contact precautions.

\section{Einleitung}

Die MRSA-Prävalenz (MRSA = Methicillin-resistenter Staphylococcus aureus) bei Aufnahme in Zentren der neurologisch-neurochirurgischen Frührehabilitation (NNFR) lag noch zu Beginn des Jahrzehnts deutlich über $10 \%$ [1], wohingegen aktuellere multizentrische Daten einen Rückgang auf bis zu 7\% [2] nahelegen. Dennoch ist evident, dass die Besiedelung mit MRSA nicht nur erhebliche ökonomische Relevanz in der NNFR hat [1], sondern mit Problemkeimen besiedelte Patienten auch ein schlechteres rehabilitatives Outcome aufweisen, was auf die hohe Morbiditätslast und den schlechten funktionellen Status dieser Patienten zurückzuführen sein dürfte [2-4].

Um Transmissionen zu verhindern, wird die Einzel- oder Kohortenisolierung im Krankenhausbereich empfohlen [5], zu dem medizinisch und leistungsrechtlich auch die NNFR zählt [6]. Identifizierte MRSA-Träger müssen so lange isoliert bleiben, bis eine Dokolonisierung erfolgreich war [5].

Da die MRSA-Aufnahmeprävalenzen in der NNFR deutlich höher als an einem normalen Krankenhaus sind (Selektion intensivpflichtiger Patienten mit hoher Morbiditätslast) [1, 2], kommt dem Screening zu Behandlungsbeginn eine überragende Bedeutung zu. Das Robert Koch-Institut (RKI) führt zum MRSA-Screening im Krankenhausbereich Folgendes aus: „Der Einsatz von MRSA-Screeningmaßnahmen kann zu einer Senkung nosokomialer Infektionsraten mit MRSA führen. Ohne Screening bleibt der überwiegende Teil der MRSA-besiedelten Patienten unerkannt. Beim Screening werden Abstrichuntersuchungen an definierten Prädilektionsstellen (mindestens beide vordere Nasenvorhöfe, Rachen, vorhandene Wunden; ggf. Perineum und Leiste) für MRSA-Besiedlungen durchgeführt und mikrobiologisch untersucht. Hierbei ist der kulturelle Nachweis des Erregers maßgeblich." [5] Damit betrachtet das RKI den kulturellen MRSANachweis als Golstandard, während zu PCR-basierten Screeningverfahren folgende Aussagen getroffen werden: „[Sie] bieten als zusätzliches Testverfahren den Vorteil einer erheblichen Zeitreduktion bei der Testdurchführung. Ihre Ergebnisse können als vorläufige Grundlage für abzuleitende krankenhaushygienische Konsequenzen dienen. Sie sind derzeit nicht zum Nachweis von MRSA-Infektionen und zur Kontrolle von MRSA-Dekolonisierungsmaßnahmen geeignet.“ [5]. Begründet wird diese Empfehlung zur PCR mit niedrigen positiven prädiktiven Werten (ca. 60$90 \%$ ), bei allerdings sehr guten negativen Vorhersagewerten (ca. 97-99\%) [7, 8]. Eine hohe Sensitivität der PCR (bis zu 100\%) zu Lasten einer etwas geringeren Spezifität ist in der Literatur schon früh beschrieben worden [9], was dadurch bedingt sein dürfte, dass die PCR auch positiv wird, wenn nur noch Zelldetritus (mit Erbmaterial des Erregers) und keine vitalen Bakterien mehr vorhanden sind. Den RKI-Empfehlungen folgend [5], wird bei einem positiven PCR-Ergebnis in vielen Zentren der NNFR auch eine kulturelle Bebrütung als Goldstandard durchgeführt.
Die vorliegende Arbeit wollte die Praktikabilität und Aussagekraft eines PCR-basierten Schnelltests zum Nachweis von MRSAErbgut in Nasen-Rachen-Abstrichen von NNFR-Patienten zum Aufnahmezeitpunkt untersuchen.

\section{Methodik}

Die BDH-Klinik Hessisch Oldendorf gGmbH ist eine gemeinnützige neurologische Fachklinik im südlichen Niedersachsen mit Akutversorgung (Intensivzentrum und zertifizierte Stroke Unit) sowie phasenübergreifender Rehabilitation, bis hin zur medizinisch-beruflichen Rehabilitation (insgesamt 250 Betten, davon 113 Krankenhausbetten, BAR-Phasen A bis E). Bei $n=66$ im Jahr 2013 aufgenommenen neurologisch-neurochirurgischen Frührehabilitanden (BAR-Phase B) wurden bei Aufnahme die MRSA-PCR aus einem Nasen-Rachen-Abstrich (BD MAX MRSA XT, BD Diagnostics, Heidelberg, Deutschland) und nachfolgend, innerhalb von maximal sieben Tagen (Eingangsdatum im Labor), eine kulturelle Untersuchung auf MRSA initiiert. Das mittlere zeitliche Intervall zwischen PCR und Kultur betrug 1,9 $( \pm 2,2)$ Tage, bei einer Spannbreite von 0 bis 7. Darunter befanden sich $n=39$ Fälle, in denen die kulturelle Untersuchung innerhalb von einem Tag nach der PCR begonnen wurde. In dieser Subgruppe ging in n= 27 Fällen noch am gleichen Tag die zur kulturellen Bebrütung vorgesehene Probe im Labor ein, in $n=12$ Fällen am Folgetag.

Zunächst erfolgte die Bebrütung der Abstriche für mindestens 18 Stunden auf chromogenen MRSA 2 Agar (Oxoid Ltd., Hampshire, Großbritannien) bei $37^{\circ} \mathrm{C}$. Verdächtige Kolonien wurden mittels Katalase, Staphytekt Plus-Test (Oxoid Ltd., Hampshire, Großbritannien) sowie dem Vitek-Resistenzsystem (bioMérieux Deutschland $\mathrm{GmbH}$, Nurtingen, Deutschland) identifiziert. Bei MRSA-positiven Befunden erfolgte eine zusätzliche Bestätigung durch den molekulargenetischen Nachweis des mec-A-Gens mittels Real-time PCR (In-House-Test).

Ermittelt wurden folgende statistische Werte:

Die Sensitivität (auch Richtig-Positiv-Rate, Empfindlichkeit oder Trefferquote; engl. sensitivity, true positive rate, recall oder hit rate) wurde berechnet als der Anteil der in der PCR als korrektpositiv identifizierten Patienten, bezogen auf die Gesamtheit der tatsächlich Positiven, also der in der Kultur identifizierten MRSAbesiedelten Patienten. Die Falsch-Negativ-Rate (engl. false negative rate oder miss rate) gibt den Anteil der in der PCR fälschlich als negativ klassifizierten an der Gesamtheit der tatsächlich positiven Patienten (in der Kultur) an.

Die Spezifität (auch Richtig-Negativ-Rate; engl. specificity, true negative rate oder correct rejection rate) gibt den Anteil der korrekt als negativ identifizierten Patienten an der Gesamtheit der in Wirklichkeit negativen an (mittels Kultur bestimmt). Die FalschPositiv-Rate (auch Ausfallrate; engl. fallout oder false positive rate) 
bezeichnet den Anteil der fälschlich in der PCR als positiv klassifizierten Patienten, die sich in Wirklichkeit (in der Kultur) als negativ erwiesen.

Der positive Vorhersagewert (auch Relevanz, Genauigkeit, positiver prädiktiver Wert; engl. precision oder positive predictive value; Abkürzung: PPW) gibt den Anteil der mittels PCR korrekt als positiv identifizierten Patienten an, geteilt durch die Summe der richtig Positiven und falsch Positiven.

Der negative Vorhersagewert (auch Segreganz oder Trennfähigkeit; engl. negative predictive value; Abkürzung: NPW) hingegen bezeichnet den Anteil der korrekt in der PCR als negativ getesteten, geteilt durch die Summe der richtig und falsch als negativ klassifizierten Patienten.

\section{Ergebnisse}

\section{Zeitdauer von maximal sieben Tagen zwischen PCR und Kultur}

Die Ergebnisse der PCR und der kulturellen Testung innerhalb von sieben Tagen sind in $>$ Tab. 1 (Kontingenztafel bzw. Wahrheitsmatrix) wiedergegeben. Die Sensitivität der PCR (im Vergleich zur Kultur) lag bei 84,6\% (11/13). Die Falsch-Negativ-Rate betrug 15,4\% (2 / 13). Die Spezifität der PCR belief sich auf 86,8\% (46/ 53), die Falsch-Positiv-Rate dementsprechend auf 13,2\% (7/ 53). Der positive Vorhersagewert (PPW) lag bei 61,1\% (11/18), der negative Vorhersagewert (NPW) bei 95,8 \% (46/48).

\section{Zeitdauer von einem Tag zwischen PCR und Kultur}

Die Ergebnisse der PCR und der kulturellen Testung innerhalb von einem Tag sind in $>$ Tab. 2 wiedergegeben. Die Sensitivität der PCR lag bei $100 \%$ (8/8). Die Falsch-Negativ-Rate betrug $0 \%(0 /$ 8). Die Spezifität der PCR belief sich auf 90,3\% (28/31), die Falsch-Positiv-Rate auf 9,7\% (3/31). Der positive Vorhersagewert (PPW) lag bei 72,7\% (8/11), der negative Vorhersagewert (NPW) bei $100 \%(28 / 28)$.

\section{Diskussion}

Multiresistente Erreger wie MRSA sind in der NNFR medizinisch und ökonomisch ein hochrelevantes Problem [1-4, 10]. Eine MRSABesiedlung führt zu einer deutlichen Verweildauerverlängerung, und betroffene Patientinnen und Patienten werden länger als die

- Tab. 1 Kontingenztafel (Wahrheitsmatrix) der Testergebnisse der PCR im Vergleich zu der kulturellen Untersuchung innerhalb von sieben Tagen.

\begin{tabular}{|l|l|l|l|l|}
\hline & \multicolumn{3}{|c|}{ Kultureller Befund } \\
\hline & & Positiv & Negativ & Summe \\
\hline & & & & \\
\hline & & & & \\
\hline & PCR & 11 & 7 & 18 \\
\hline & Positiv & 2 & 46 & 48 \\
\hline & Segativ & 13 & 53 & 66 \\
\hline
\end{tabular}

- Tab. 2 Kontingenztafel (Wahrheitsmatrix) der Testergebnisse der PCR im Vergleich zu der kulturellen Untersuchung innerhalb von einem Tag.

\begin{tabular}{|c|c|c|c|c|}
\hline & & \multicolumn{2}{|c|}{ Kultureller Befund } & \multirow[b]{2}{*}{ Summe } \\
\hline & & Positiv & Negativ & \\
\hline \multirow[t]{3}{*}{ PCR } & Positiv & 8 & 3 & 11 \\
\hline & Negativ & 0 & 28 & 28 \\
\hline & Summe & 8 & 31 & 39 \\
\hline
\end{tabular}

Hälfte ihres Aufenthaltes isoliert [1], was - zusätzlich zu einem ohnehin schlechteren Outcome - die Rehabilitation erschwert [1, 3, 4]. Daher ist es wichtig, MRSA-Träger gleich zu Beginn der NNFR zuverlässig zu identifizieren, um eine Transmission zu verhindern [5]. Andererseits will man den kritisch kranken Patientinnen und Patienten unnötige und belastende Isolierungsmaßnahmen ersparen [1]. Zudem gibt es Einrichtungen, die Betroffene mit entsprechenden Risikofaktoren bis zum Vorliegen eines negativen Befunds isolieren. Durch die PCR, die i.d. R. schon nach wenigen Stunden vorliegt, kann damit auch die Isolationsdauer nach Aufnahme deutlich verkürzt werden. Für einen MRSA-Fall in der neurologisch-neurochirurgischen Frührehabilitation wurden die indirekten Kosten allein durch die Isolierung auf $12.800 €$ geschätzt, die durch das aktuelle Fallpauschalen-System nicht kompensiert werden [1]. In einer anderen Untersuchung wurden die Mehrkosten durch multiresistente Erreger in der neurologischen Rehabilitation, u.a. wegen der notwendigen Isolierung, auf $418 €$ pro Tag und Patient geschätzt [10].

Als kostengünstiges und zeitsparendes Verfahren, um eine MRSA-Kolonisation im Nasen-Rachen-Raum zu detektieren, hat sich die MRSA-PCR bewährt [9]. Bei hoher Sensitivität ist die Spezifität etwas geringer [9], sodass mit falsch-positiven Ergebnissen gerechnet werden muss. In der vorliegenden Studie sollte die Praktikabilität und Validität der Methode in der NNFR untersucht werden. Dabei ergab sich für die PCR eine Sensitivität von $84,6 \%$, bei einer Spezifität von 86,8\%. Diese Werte bezogen sich auf den Vergleich der PCR mit einer innerhalb von sieben Tagen initiierten kulturellen Untersuchung. Die im Vergleich zu der in der Literatur berichteten niedrigeren Sensitivität [9] mag durch diese zeitliche Verzögerung bedingt sein. Auch der positive prädiktive Wert (PPW) war mit nur 61,1\% deutlich niedriger, als Voruntersuchungen dies nahelegen [7, 8], bei allerdings hohem negativen prädiktiven Wert (NPW) mit 95,8\%. Bei immerhin 39 Frührehabilitanden konnte das Intervall zwischen PCR und nachfolgender Kultur auf einen Tag reduziert werden. Hierdurch stiegen die Sensitivität auf $100 \%$ und die Spezifität auf 90,3\%. In dieser Subgruppe betrug der PPW 72,7\%, der NPW $100 \%$. Die Ergebnisse zeigen, dass nach einem positiven PCR-Befund rasch (innerhalb von 24 Stunden) eine kulturelle Untersuchung durchgeführt werden sollte, um eine MRSA-Besiedelung zu verifizieren und falsch-positive Ergebnisse ausschließen zu können. Bis zum Vorliegen der kulturellen Befunde sollte der Betroffene isoliert bleiben [5]. Insbesondere der hohe NPW (95,8 bzw. $100 \%$ ) zeigt jedoch an, dass negativ getestete 
Patientinnen und Patienten auch mit hoher Wahrscheinlichkeit keine MRSA-Träger sind. Hierdurch ist die in vielen NNFR-Kliniken angewendete Praxis zu rechtfertigen, bei negativem PCR-Ergebnis auf eine Isolierung zu verzichten.

\section{Fazit für die Praxis}

Die Besiedelung mit MRSA stellt eine große Herausforderung in der neurologisch-neurochirurgischen Frührehabilitation (NNFR) dar, daher muss bereits zu Beginn der Behandlung eine Kolonisierung mit diesem Keim ausgeschlossen werden. Dies gelingt mit hoher Sensitivität (bis zu 100\%) bei etwas geringerer Spezifität mittels PCR-basierter Tests. Ein hoher negativer Vorhersagewert der PCR spricht dafür, dass bei negativem Testergebnis auf Isolierungsmaßnahmen verzichtet werden kann.

Interessenkonflikt

Die Autoren deklarieren keine Interessenkonflikte.

\section{Literatur}

[1] Rollnik JD, Samady M, Grüter L. Multiresistente Erreger in der neurologisch-neurochirurgischen Frührehabilitation (2004-2013). Rehabilitation (Stuttg) 2014; 53: 346-350.

[2] Rollnik JD, Bertram M, Bucka C et al. Outcome of neurological early rehabilitation patients carrying multi-drug resistant bacteria: results from a German multi-center study. BMC Neurol 2017; 17: 53.
[3] Rollnik JD. Outcome of MRSA carriers in neurological early rehabilitation. BMC Neurol 2014; 14: 34.

[4] Rollnik JD. Outcome of neurological early rehabilitation patients colonized with extended-spectrum beta-lactamase (ESBL) producing bacteria. Open J Therapy Rehabil 2015; 3: 1-8.

[5] Kommission für Krankenhaushygiene und Infektionsprävention am RKI (KRINKO). Empfehlungen zur Prävention und Kontrolle von Methicillinresistenten Staphylococcus aureus-Stämmen (MRSA) in medizinischen und pflegerischen Einrichtungen. Bundesgesundheitsbl 2014; 57: 696-732.

[6] Rollnik JD, Platz T, Böhm KD et al. Argumente für eine Zuordnung der neurologisch-neurochirurgischen Frührehabilitation (Phase B) zum Krankenhausbereich ( 339 SGB V). Positionspapier der Kliniken des BDH Bundesverband Rehabilitation. Akt Neurol 2011; 38: 362-368.

[7] Daeschlein G, Assadian O, Daxboeck F et al. Multiplex PCR-ELISA for direct detection of MRSA in nasal swabs advantageous for rapid identification of non-MRSA carriers. Eur J Clin Microbiol Infect Dis 2006; 25 : 328-330.

[8] Holfelder M, Eigner U, Turnwald AM et al. Direct detection of methicillin-resistant Staphylococcus aureus in clinical specimens by a nucleic acid-based hybridisation assay. Clin Microbiol Infect 2006; 12: 11631167.

[9] Cavassini M, Wenger A, Jaton K et al. Evaluation of MRSA-Screen, a simple anti-PBP 2a slide latex agglutination kit, for rapid detection of methicillin resistance in Staphylococcus aureus. J Clin Microbiol 1999; 37: 1591-1594.

[10] Roukens R, Lauster F, Bara M et al. Mehrkosten durch multiresistente Erreger in der Neurorehabilitation. Bundesgesundheitsblatt Gesundheitsforschung Gesundheitsschutz 2017; 60: 1075-1082. 